\title{
An Efficient Automatic Test Generation System for Path Delay Faults in Combinational Circuits
}

\author{
Ananta K. Majhi and James Jacob \\ Dept. of Electrical Comm. Eng. \\ Indian Institute of Science \\ Bangalore 560 012, India \\ majhi@ece.iisc.ernet.in, james@ece.iisc.ernet.in
}

\author{
Lalit M. Patnaik \\ Microprocessor Applications Lab. \\ Indian Institute of Science \\ Bangalore 560 012, India \\ lalit@micro.iisc.ernet.in
}

\author{
Vishwani D. Agrawal \\ AT\&T Bell Laboratories \\ 600 Mountain Avenue \\ Murray Hill, NJ 07974 \\ va@research.att.com
}

\begin{abstract}
The new test pattern gerieration system for path delay faults in combinational logic circuits considers robust and nonrobust tests, simultaneously. Once a robust test is obtained for a path with a given transition, another test for the same path with the opposite transition is immediately derived with a small extra effort. To facilitate the simultaneous consideration of robust and nonrobust tests, we derive a new nine-value logic system. An efficient multiple backtrace procedure satisfies test generation objectives. We also use a path selection method which covers all lines in the logic circuit by the longest and the shortest possible paths through them. A fault simulator in the system gives information on robust and nonrobust detection of faults either from a given target set or all path faults. Experimental results on ISCAS'85 and ISCAS'89 benchmark circuits substantiate the efficiency of our algorithm in comparison to other published results.
\end{abstract}

\section{Introduction}

The path delay fault model [16] is extensively used since it represents the influence of distributed delays on the operation of clocked systems. The complexity of test generation for delay faults is rather high due to factors such as the large number of possible paths, large number of tests, and excessive computation time for test generation. In this paper, we present a novel test generation algorithm capable of efficiently generating robust and nonrobust tests for path delay faults.

Most of the delay fault testing methods reported hitherto $[3,10]$ are based on the relatively simple concept of the PODEM algorithm [7]. Fuchs et al use an extended multiple backtrace procedure and the concept of static and dynamic learning for path delay fault testing [5]. Non-enumerative methods for test generation and fault simulation of delay faults have recently appeared in the literature $[9,14]$.

Our method, uses a multiple backtrace procedure similar to that of the FAN algorithm [6] for satisfying the test generation objectives. The new ideas incorporated in our algorithm are as follows:

1. A new 9-value logic system to derive robust and nonrobust tests for path delay faults (Section 2).
2. A multiple backtrace procedure finds and resolves conflicts at internal nodes rather than at PIs. This is illustrated by Example 1.

3. Direct derivation of a test for the opposite transition once a test for a path for a given transition is found (Examples 2 and 3 ).

4. Path delay fault simulation is done immediately following the generation of a test to determine robust and nonrobust detection status of other faults.

5. Tests are generated for all paths or, especially in case of too many paths, for one longest and one shortest path per line (Section 3).

\section{A Nine-Value Logic System}

Multi-valued logic has been used by practically all researchers for test generation and fault simulation of path delay faults. In a recent paper, Bose et al [2] derive a 23-value logic system for the test generation for multiple paths. Fuchs et al [5] introduce a 10valued logic for robust and a three-valued logic for nonrobust test generation.

Our goal is to derive an optimal logic system that will allow simultaneous consideration of robust and nonrobust tests. In earlier literature $[2,5]$, multivalued logic system has been derived by manipulating three $(0,1$ and $X)$ logic states which are simultaneously considered in two consecutive time frames (initial, final) of the clock period. In this work, we manipulate the 0,1 and $X$ logic states for three (initial, intermediate and final) intervals within two clock periods. Thus, we get $3^{3}=27$ possible transition states which are given in Table 1 . These 27 states eventually collapse to a 9-value logic system.

The transition states 1-4 and 10-13 of Table 1 in which the logic values are fully specified are represented by unique values, i.e., (S0, G0, FT) and (S1, $\mathrm{G} 1, \mathrm{RT}$ ) respectively. The composite states (transition states 5-9 and 14-18) are absorbed together and are represented by X0 and X1 respectively. The last 9 states (19-27) are collapsed to a single logic value XX since the final logic value is $\mathrm{X}$ (don't care). Signal values G0 and G1 represent static hazards (i.e., glitches 
Table 1: Possible logic states

\begin{tabular}{|c|c|c|c|}
\hline $\begin{array}{l}\text { State } \\
\text { id }\end{array}$ & $\begin{array}{c}\text { Possible } \\
\text { states }\end{array}$ & $\begin{array}{c}\text { Fully } \\
\text { specified }\end{array}$ & $\begin{array}{c}\text { Composite } \\
\text { states }\end{array}$ \\
\hline 1 & 000 & $\{\mathrm{~S} 0\}$ & - \\
\hline 2 & 010 & $\{\mathrm{G} 0\}$ & - \\
\hline 3 & 100 & $\{\mathrm{FT}\}$ & - \\
\hline 4 & 110 & $\{\mathrm{FT}\}$ & - \\
\hline 5 & $0 \times 0$ & - & $\{\mathrm{S} 0, \mathrm{G} 0\}$ \\
\hline 6 & $X 00$ & - & $\{\mathrm{S} 0, \mathrm{FT}\}$ \\
\hline 7 & X X 0 & - & $\{\mathrm{S} 0, \mathrm{G} 0, \mathrm{FT}\}$ \\
\hline 8 & $1 \times 0$ & - & $\{\mathrm{FT}, \mathrm{FT}\}$ \\
\hline 9 & $X 10$ & - & $\{\mathrm{G} 0, \mathrm{FT}\}$ \\
\hline 10 & 111 & $\{\mathrm{~S} 1\}$ & - \\
\hline 11 & 101 & $\{\mathrm{G} 1\}$ & - \\
\hline 12 & 011 & $\{\mathrm{RT}\}$ & - \\
\hline 13 & 001 & $\{\mathrm{RT}\}$ & - \\
\hline 14 & $0 \times 1$ & - & $\{\mathrm{RT}, \mathrm{RT}\}$ \\
\hline 15 & $1 \mathrm{X} 1$ & - & $\{\mathrm{S} 1, \mathrm{G} 1\}$ \\
\hline 16 & X 01 & - & $\{\mathrm{RT}, \mathrm{G} 1\}$ \\
\hline 17 & X X 1 & - & $\{\mathrm{S} 1, \mathrm{G} 1, \mathrm{RT}\}$ \\
\hline 18 & $X 11$ & 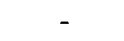 & $\{\mathrm{RT}, \mathrm{S} 1\}$ \\
\hline 19 & $00 X$ & - & - \\
\hline 20 & $11 X$ & - & - \\
\hline 21 & $X 0 X$ & - & - \\
\hline 22 & $X \perp X$ & - & - \\
\hline 23 & $0 X X$ & - & - \\
\hline 24 & $1 \mathrm{XX}$ & - & - \\
\hline 25 & $01 \mathrm{X}$ & - & - \\
\hline 26 & $10 X$ & - & - \\
\hline 27 & X X X & - & - \\
\hline
\end{tabular}

0-1-0 and 1-0-1) and these are explicitly used for generation of nonrobust tests. More details on generating nonrobust tests will be given in a following section. We believe that our 9 -value logic will be the minimum required for the simultaneous consideration of robust and nonrobust tests. In Table 2, we have given the signal value representation and their corresponding logic values in both vectors.

\section{Test Generation}

In most practical cases, the large number of paths makes it impossible to consider all path faults for the purpose of test generation and fault simulation. There are several methods available in the literature for the selection of a subset of paths [13]. Recently, Pomeranz et al [14] and Heragu [9] have described methods without enumerating paths. In recent papers $[8,11]$, it has been shown that the determination of an optimal clocking period highly depends on the accuracies of the estimated longest path length and the shortest path length in the circuit. In this work, we have introduced a method for selecting a subset of paths which covers all lines in the logic circuit at least once and includes the shortest (S) as well as the longest (L) possible path through each line.
Table 2: Signal value representation

\begin{tabular}{|cccl|}
\hline $\begin{array}{c}\text { Name of } \\
\text { signal value }\end{array}$ & $\begin{array}{c}\text { Initial } \\
\text { value }\end{array}$ & $\begin{array}{c}\text { Final } \\
\text { value }\end{array}$ & $\begin{array}{l}\text { Hazard } \\
\text { conditions }\end{array}$ \\
\hline S0 & 0 & 0 & no static hazard \\
X0 & $\mathrm{X}$ & 0 & hazard possible \\
FT & 1 & 0 & no dynamic hazard \\
S1 & 1 & 1 & no static hazard \\
X1 & $\mathrm{X}$ & 1 & hazard possible \\
RT & 0 & 1 & no dynamic hazard \\
XX & $\mathrm{X}$ & $\mathrm{X}$ & hazard possible \\
G0 & 0 & 0 & static hazard $(0-1-0)$ \\
G1 & 1 & 1 & static hazard $(1-0-1)$ \\
\hline
\end{tabular}

First, we trace the circuit from POs to PIs in a breadth-first manner for assigning a label depth to each line $L$ which represents the length (in terms of logic levels) of the longest path from $L$ to any PO. Next, for selecting and enumerating the longest paths through each line, we do a depth-first trace from each PI toward POs along the lines with maximum depth. For each intermediate line which is not covered by the longest paths, we continue backward trace toward PIs along the line with maximum level and forward trace along the line with maximum depth. Similarly, for selecting the shortest paths, we trace the circuit in both directions (i.e., from $\mathrm{PI}$ to $\mathrm{POs}$ and vice versa) for assigning two labels [depth $h_{p i}$, dept $\left.h_{p o}\right]$ to each line $L$ which represent the minimum length (in terms of logic levels) from $L$ to any $\mathrm{PI}$ and PO. We continue the backward and forward trace along the minimum depth lines for selecting the shortest paths.

Our test generation method uses a multiplebacktrace procedure similar to that of the FAN algorithm [6].

First we do an implication with respect to the transition (rising or falling) at the input of the path and other inputs unspecified (XX). The off-path inputs of the gates along the target path are then set to suitable logic values in order to propagate the transition. Next, we backtrace from the primary outputs towards the primary inputs in a breadth-first manner in order to satisfy the objectives. During the backtrace operation, if we create a new objective on an input of a gate $G$ and this input happens to be a fanout branch, then we immediately create another objective with the same logic value on the fanout stem. After doing an implication we mark all fanout branches as tried. Hence, before setting an objective on any fanout branch during backtrace, we check whether or not it has already been tried by previous objectives. The procedure for test generation is illustrated by the following example.

Example 1: Consider the circuit in Figure 1. A test is to be generated for the rising transition on path 4-57-8-10-12. During implication the fanout branches 5 , 6,8 and 9 will be marked as assigned. The primary objectives obtained are $(2=\mathrm{S} 1,3=\mathrm{X} 1$ and $11=\mathrm{X} 1)$. During backtrace the last level objective $(11=\mathrm{X} 1)$ will not create any new objective since both fanins (6 and 9 ) 
are already marked as assigned. The other two objectives are fanout branches of stem 1 . Hence, after intersection of the two logic values $(S 1 \cap X 1=S 1)$, S1 will be assigned to the stem 1, which is a PI. Thus, after simulation we find a robust test $(1=\mathrm{S} 1,4=\mathrm{RT})$, since all primary objectives are satisfied.

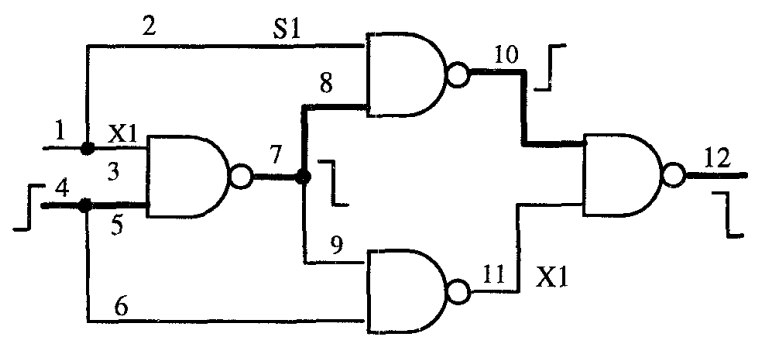

Figure 1: Example of multiple backtrace

Once we find a robust test for a transition on a target path, instead of generating a completely new test for the opposite transition we derive the second test by replacing $\mathrm{X} 1(\mathrm{X} 0)$ with steady values $\mathrm{S} 1(\mathrm{~S} 0)$ in the already generated test and reversing the transition at the input of the target path. The underlying principle is that since the second vector is a test for a stuck fault on the PI at the source of the path, the opposite transition will always reach the destination of the path if the sensitizing condition remains unchanged. In most cases this modified vector pair will be a valid robust/nonrobust test for the opposite transition. If the implied logic values are a subset ( $S 0$ and $G 0 \subset X 0 ; S 1$ and $G 1 \subset X 1$ ) of the primary objective logic values or are the same, then the derived test will be a robust test. The following example illustrates such derivation.

Example 2: Consider the circuit given in Figure 2, where a test is generated for the rising transition on path 1-2-10-12. The primary objectives to be justified are $8=\mathrm{X} 1$ and $11=\mathrm{S} 1$. Following Example 1, we obtain a robust test, i.e., $1=\mathrm{RT}$ and $4=\mathrm{S} 0$. The new set of primary objectives for the opposite transition on the same path $(1-2-10-12)$ will be $8=S 1$ and $11=\mathrm{X} 1$. The new test is obtained only by reversing the transition on line 1 ( $1=\mathrm{FT}$ and $4=\mathrm{S} 0$ ) since input 4 has a steady value S0. After implication, we find that the implied logic value on line 11 is $\mathrm{S} 1$ which is a subset of X1 (i.e., primary objective logic value on line 11). Hence, we find a robust test for the opposite transition with minimal effort.

In some cases, the test derived for the opposite transition as described above may not be a robust test. If at least one of the primary objective logic values is $\mathrm{S} 1$ (S0) and the implied logic value becomes $\mathrm{G1}(\mathrm{G} 0)$, then the derived test will be a nonrobust test for the opposite transition. The following example illustrates the derivation of a nonrobust test.

Example 3: In Example 1, suppose we have to find a test for the opposite transition (i.e., falling) on the same path (4-5-7-8-10-12). The new set of primary objectives will be $2=\mathrm{X} 1,3=\mathrm{S} 1$ and $11=\mathrm{S} 1$. The test

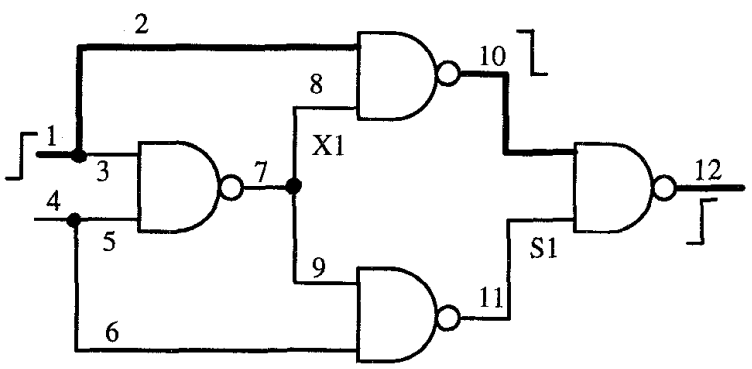

Figure 2: Robust test for opposite transition

will be obtained by reversing the transition on line 4 $(1=\mathrm{S} 1,4=\mathrm{FT})$ since input 1 has a steady value $\mathrm{S} 1$. After implication, we find that the implied logic value of line 11 is $\mathrm{G} 1$, whereas its primary objective logic value is $\mathrm{S} 1$. Hence, the derived test will be a nonrobust test for the falling transition. In such situations, a fresh attempt will be made to generate a robust test for the given path and transition.

\section{Results}

We have implemented the proposed path delay test generation algorithm in the $\mathrm{C}$ language (about 3000 lines of code) on an IBM RS-6000/580 workstation. In order to benchmark and demonstrate the efficiency of our test generator, we have performed ATG of robust and nonrobust tests on the ISCAS'85 and ISCAS' 89 benchmark circuits.

Table 3 gives the results for ISCAS' 85 benchmarks. Path faults is the number of logical paths considered for test generation. This is twice the number of the physical paths selected to cover each line via the longest (L) path through it. For the purpose of comparison, we have also given the test generation results when the path selection procedure is modified to choose the shortest (S) path through each line. As expected, in general, the coverage is higher for the shorter paths. We have integrated a fault simulator [12] in the system. Once a robust or nonrobust test is generated, immediately we do fault simulation with respect to the test vector. Both robust and nonrobust detection of path delay faults are reported. Robustly detected paths are then marked in the targeted path list and hence not considered for further test generation. Third and forth columns of Table 3 give the number of robust and nonrobust tests generated by the test generator within the backtrack limit of 100 . Fifth and sixth columns give the number of path faults which are robustly and nonrobustly tested from the targeted fault list while columns seven and eight are for the non-targeted faults. The CPU time (in seconds) is given for the complete ATPG process. For circuit c6288, the number of nonrobustly detected paths was extremely large even for small number of tests and it requires large amount of CPU time and memory for successful completion. Hence, we have not considered the nonrobust detection of paths during simulation.

Our results in Table 3 may be compared to two earlier works $[14,15]$ which give test generation re- 
Table 3: Delay test results for ISCAS'85 benchmarks

\begin{tabular}{|c|c|c|c|c|c|c|c|c|}
\hline \multirow[t]{2}{*}{ Circuit } & \multirow{2}{*}{$\begin{array}{r}\text { Path } \\
\text { faults }\end{array}$} & \multicolumn{2}{|c|}{$\begin{array}{c}\text { Tests } \\
\text { generated }\end{array}$} & \multicolumn{2}{|c|}{$\begin{array}{l}\text { Paths detected } \\
\text { (Targeted) }\end{array}$} & \multicolumn{2}{|c|}{$\begin{array}{l}\text { Paths detected } \\
\text { (Non-targeted) }\end{array}$} & \multirow[b]{2}{*}{ CPU s* } \\
\hline & & Rob. & Nrob. & Rob. & Nrob. & Rob. & Nrob. & \\
\hline \multirow[t]{2}{*}{$\mathrm{c880}$} & $744(\mathrm{~L})$ & 469 & 0 & 520 & 5 & 244 & 711 & 15.45 \\
\hline & $744(\mathrm{~S})$ & 620 & 3 & 712 & 8 & 41 & 439 & 9.08 \\
\hline \multirow[t]{2}{*}{ c1355 } & $1100(\mathrm{~L})$ & 0 & 0 & 0 & 0 & 0 & 0 & 39.31 \\
\hline & $1100(\mathrm{~S})$ & 16 & 0 & 26 & 0 & 28 & 34 & 85.12 \\
\hline \multirow[t]{2}{*}{ c1908 } & $1286(\mathrm{~L})$ & 57 & 1 & 83 & 13 & 24 & 902 & 177.93 \\
\hline & $1286(\mathrm{~S})$ & 319 & 0 & 366 & 61 & 37 & 1221 & 114.37 \\
\hline \multirow[t]{2}{*}{ c2670 } & $2046(\mathrm{~L})$ & 572 & 9 & 589 & 59 & 498 & 1897 & 249.31 \\
\hline & $2046(S)$ & 944 & 4 & 1031 & 61 & 218 & 4598 & 139.20 \\
\hline \multirow[t]{2}{*}{ c3540 } & $2584(\mathrm{~L})$ & 68 & 0 & 87 & 2 & 113 & 2436 & 579.11 \\
\hline & $2584(\mathrm{~S})$ & 541 & 0 & 683 & 88 & 1017 & 7999 & 554.83 \\
\hline \multirow[t]{2}{*}{ c5315 } & $4404(\mathrm{~L})$ & 2242 & 26 & 2245 & 433 & 2220 & 26138 & 808.30 \\
\hline & $4404(\mathrm{~S})$ & 2183 & 4 & 2889 & 198 & 787 & 17275 & 616.98 \\
\hline \multirow[t]{2}{*}{ c6288 } & $4832(\mathrm{~L})$ & 27 & 0 & 34 & NA & 8 & NA & 1198.57 \\
\hline & $4832(\mathrm{~S})$ & 195 & 52 & 213 & $\mathrm{NA}$ & 102 & NA & 2401.01 \\
\hline \multirow[t]{2}{*}{ c7552 } & $5480(\mathrm{~L})$ & 846 & 64 & 953 & 578 & 1968 & 28370 & 2083.40 \\
\hline & $5480(\mathrm{~S})$ & 2095 & 35 & 2502 & 330 & 5023 & 25345 & 2257.43 \\
\hline
\end{tabular}

* IBM RS-6000/580

Table 4: Test results for scan/hold versions of ISCAS' 89 benchmarks

\begin{tabular}{|c|c|c|c|c|c|c|c|c|c|c|c|}
\hline \multirow[b]{2}{*}{ Circuit } & \multirow[b]{2}{*}{$\begin{array}{r}\text { Path } \\
\text { faults }\end{array}$} & \multicolumn{4}{|c|}{ Our ATPG } & \multicolumn{2}{|c|}{ [1] } & \multicolumn{2}{|c|}{ [3] } & \multicolumn{2}{|c|}{ [4] } \\
\hline & & $\begin{array}{r}\text { Rob./nrob. } \\
\text { tests }\end{array}$ & $\begin{array}{l}\text { Rob. } \\
\text { det. }\end{array}$ & $\begin{array}{l}\text { Nrob. } \\
\text { det. }\end{array}$ & $\begin{array}{r}\mathrm{CPU} \\
\text { s† }\end{array}$ & $\begin{array}{l}\text { Paths } \\
\text { det. }\end{array}$ & $\begin{array}{r}\mathrm{CPU} \\
\mathrm{S} * *\end{array}$ & $\begin{array}{l}\text { Paths } \\
\text { det. }\end{array}$ & $\begin{array}{r}\mathrm{CPU} \\
\text { st }\end{array}$ & $\begin{array}{c}\text { Paths } \\
\text { det. }\end{array}$ & $\begin{array}{r}\mathrm{CPU} \\
\mathrm{s} \ddagger\end{array}$ \\
\hline s27 & 56 & $28 / 0$ & 50 & 0 & 0.03 & 50 & 0.0 & 50 & 0.1 & 50 & 0.0 \\
\hline $\mathrm{s} 208$ & 290 & $232 / 0$ & 288 & 0 & 0.49 & 290 & 0.2 & 290 & 2.7 & 290 & 4.9 \\
\hline s298 & 462 & $221 / 1$ & 328 & 22 & 1.24 & 343 & 0.2 & 331 & 4.9 & 343 & 5.1 \\
\hline s 344 & 710 & $363 / 4$ & 559 & 45 & 2.07 & 611 & 0.6 & 602 & 8.6 & 611 & 23.4 \\
\hline s349 & 730 & $361 / 4$ & 554 & 49 & 2.02 & 611 & 0.6 & 602 & 9.0 & 611 & 14.6 \\
\hline s382 & 800 & $349 / 13$ & 660 & 65 & 2.02 & 667 & 0.5 & 664 & 14.1 & 667 & 21.6 \\
\hline s.386 & 414 & $210 / 0$ & 406 & 2 & 0.71 & 413 & 0.3 & - & - & - & - \\
\hline $\mathrm{s} 400$ & 896 & $348 / 5$ & 646 & 59 & 2.59 & 665 & 0.6 & - & - & - & - \\
\hline $\mathrm{s} 420$ & 738 & $612 / 0$ & 736 & 0 & 2.04 & 738 & 1.3 & 738 & 13.8 & 738 & 34.7 \\
\hline $\mathrm{s} 444$ & 1070 & $376 / 60$ & 579 & 190 & 3.69 & 586 & 0.8 & 513 & 31.7 & 586 & 13.9 \\
\hline s 510 & 738 & $391 / 2$ & 723 & 14 & 2.20 & 729 & 0.9 & 703 & 11.9 & 729 & 50.2 \\
\hline s526 & 820 & $419 / 2$ & 651 & 43 & 3.03 & 694 & 0.5 & - & - & 694 & 41.8 \\
\hline s526n & 816 & $420 / 2$ & 652 & 40 & 3.52 & 695 & 0.5 & - & - & 695 & 38.4 \\
\hline s641 & 3488 & $1302 / 49$ & 1955 & 168 & 28.58 & 1979 & 8.5 & - & - & 1979 & 275.6 \\
\hline $\mathrm{s} 820$ & 984 & $554 / 0$ & 964 & 7 & 5.62 & 980 & 1.1 & 961 & 18.3 & 980 & 122.0 \\
\hline s832 & 1012 & $546 / 0$ & 966 & 11 & 6.70 & 984 & 1.3 & 971 & 19.8 & 984 & 125.5 \\
\hline s838 & 2018 & $1756 / 0$ & 2016 & 0 & 13.19 & 2018 & 9.7 & - & - & 2018 & 496.4 \\
\hline s953 & 2266 & $985 / 0$ & 2190 & 32 & 14.61 & 2256 & 4.1 & 2221 & 44.2 & 2302 & 256.6 \\
\hline s1196 & 6194 & $1748 / 7$ & 3408 & 119 & 252 & 3579 & 13.9 & 2938 & 282.0 & 3581 & 1146 \\
\hline $\mathrm{s} 1238$ & 7118 & $1656 / 3$ & 3450 & 42 & 275 & 3587 & 14.0 & - & - & 3589 & 1253 \\
\hline $\mathrm{s} 1488$ & 1924 & $805 / 1$ & 1830 & 35 & 20.79 & 1875 & 3.1 & 1830 & 72.3 & 1875 & 315.5 \\
\hline s1494 & 1952 & $801 / 1$ & 1837 & 37 & 21.84 & 1882 & 3.1 & 1838 & 75.7 & 1882 & 304.5 \\
\hline $\mathrm{s} 5378$ & 27062 & $8341 / 83$ & 14421 & 1662 & 3236 & 18656 & 98.0 & 16222 & 26600 & 6554 & 8450 \\
\hline $\mathrm{s} 713$ & $480 *$ & $146 / 4$ & 183 & 1 & 9.70 & 1184 & 22.0 & - & - & 232 & 50.0 \\
\hline $\mathrm{s} 1423$ & $1172 *$ & $677 / 0$ & 893 & 19 & 38.1 & 28696 & 436.3 & - & - & 2730 & 1300 \\
\hline s9234 & $5284 *$ & $1913 / 161$ & 2549 & 403 & 1241 & 21389 & 481.3 & - & - & 231 & 1050 \\
\hline $\mathbf{s 1 3 2 0 7}$ & $8008 *$ & $3920 / 219$ & 5665 & 760 & 1581 & 27503 & 2649 & - & - & 133 & 2740 \\
\hline $\mathrm{s} 15850$ & $9114 *$ & $3793 / 257$ & 5146 & 763 & 2957 & - & - & - & - & 6 & 2190 \\
\hline s35932 & $28504 *$ & $11149 / 1787$ & 15033 & 4383 & 22294 & 21783 & 308.8 & - & - & 335 & 1270 \\
\hline s38417 & $23182 *$ & $12479 / 300$ & 16532 & 1196 & 19531 & - & - & - & - & 242 & 4120 \\
\hline s38584 & $30466 *$ & $18631 / 265$ & 24268 & 1146 & 28642 & 92235 & 2614 & - & - & 12 & 950 \\
\hline
\end{tabular}

* Partial set of faults † IBM RS-6000/580 ** DECstation 2000/500 † SUN SPARC2 
sults for ISCAS'85 benchmarks. However, since the number of modeled path faults and the machines used are different, a direct comparison may not be meaningful. It may be noticed that the fraction of modeled paths that are robustly tested in our method is significantly higher than that reported in $[14,15]$. The results support the merit of selecting the longest and shortest paths and the efficiency of the novel ideas in our system.

Table 4 presents the results of our algorithm for scan/hold versions of ISCAS'89 benchmark circuits. Path faults is the number of both rising and falling transitions on all possible physical paths. Under Our $A T P G$, Rob./nrob. tests is the number of robust and nonrobust tests generated within the backtrack limit of 100 . Rob. det. is the number of path faults which are robustly tested. Nrob. det. is the number of path faults that are nonrobustly tested by the fault simulator. The CPU time (in seconds), given for IBM RS$6000 / 580$ workstation, includes the time for test generation and fault simulation. For the last eight benchmarks shown in Table 4, we have considered only a subset of all possible path faults since the total number of paths in these circuits was very large. These paths were obtained by the path selection algorithm given in Section 3 and include one shortest path per line.

For comparison, we include the results of $[1,3,4]$ in Table 4 which reports the coverage of robustly tested paths and the CPU times for these three approaches. All possible path faults are modeled in $[1,3]$. Chakradhar et al [4] consider 10,000 path faults for the last nine benchmarks of Table 4 and all possible path faults for other benchmarks. For a direct comparison, CPU times must be normalized for the corresponding machine speeds. Such normalization is not done in Table 4. However, our ATPG appears to be more efficient than the techniques of $[3,4]$ and the fault coverage is comparable. The CPU times reported in [1] are better than our results. Such performance is generally expected from the binary decision diagram (BDD) method. It must be remembered, however, that the time and memory complexities of the BDD approach [1] can be impractical for some circuits.

\section{Conclusion}

We have presented a novel path delay test generation algorithm which incorporates an efficient multiple backtrace procedure for signal value justification. The nine-value logic system provides an efficient way of deriving both robust and nonrobust tests. Once we find a robust test for a path delay fault, we modify it to derive another test for the opposite transition. In most cases, the derived test becomes either a robust or a nonrobust test for the same path with opposite transition. Thus, we are able to considerably reduce the test generation time. A subset of paths is selected for test generation covering all lines in the logic circuit at least once. This subset includes the longest and shortest paths through each line. We use a fault simulator for robust and nonrobust detection of path faults.

We are currently extending this approach for mul- tiple pass test generation through longest sensitizable paths to cover all lines.

\section{References}

[1] D. Bhattacharya, P. Agrawal, and V. D. Agrawal, "Delay Fault Test Generation for Scan/Hold Circuits using Boolean Expressions", Proc. Design Autom. Conf., pp. 159-164, June 1992.

[2] S. Bose, P. Agrawal, and V. D. Agrawal, "Logic Systems for Path Delay Fault Test Generation", Proc. EURO-DAC, pp. 200-205, September 1993.

[3] S. Bose, P. Agrawal, and V. D. Agrawal, "Generation of Compact Delay Tests by Multiple Path Activation", Proc. Int'1 Test Conf., pp. 714-723, October 1993.

[4] S. T. Chakradhar, M. A. Iyer, and V. D. Agrawal, "Energy Minimization Based Delay Testing", Proc. European Conf. Design Autom. (EDAC), pp. 280-284, March 1992.

[5] K. Fuchs, F. Fink, and M. H. Schulz, "DYNAMITE: An Efficient Automatic Test Pattern Generation System for Path Delay Faults", IEEE Trans. CAD, Vol. 10, pp. 1323-1334, October 1991.

[6] H. Fujiwara and T. Shimono, "On the Acceleration of Test Generation Algorithms", IEEE Trans. Comput., Vol. C-32, pp. 1137-1144, December 1983.

[7] P. Goel, "An Implicit Enumeration Algorithm to Generate Tests for Combinational Logic Circuits", IEEE Trans. Comput., Vol. C-30, pp. 215-222, March 1981.

[8] C. T. Gray, W. Liu, and R. K. Cavin, "Timing Constraints for Wave-Pipelined Systems", IEEE Trans. CAD, Vol. 13, pp. 987-1004, August 1994.

[9] K. Heragu, "Approximate and Statistical Methods to Compute Delay Fault Coverage", Master's Thesis, Dept. of ECE, Rutgers University, New Brunswick, New Jersey, 1994

[10] C. J. Lin and S. M. Reddy, "On Delay Fault Testing in Logic Circuits", IEEE Trans. CAD, Vol. CAD-6, pp. 694-703, September 1987.

[11] L.-R. Liu, H.-C. Chen, and D. H. C. Du, "The Calculation of Signal Stable Ranges in Combinational Circuits", IEEE Trans. CAD, Vol. 13, pp. 1016-1023, August 1994.

[12] A. K. Majhi, J. Jacob, and L. M. Patnaik, "A Novel Path Delay Fault Simulator using Binary Logic", to appear in VLSI Design: An Int'l Jour. Custom-Chip Design, Simulation and Testing, 1994.

[13] S. Patil and S. M. Reddy, "A Test Generation System for Path Delay Faults", Proc. Int'l Conf. Computer Des., pp. 40-43, 1989.

[14] I. Pomeranz, S. M. Reddy, and P. Uppaluri, "NEST: A Non-Enumerative Test Generation Method for Path Delay Faults in Combinational Circuits", Proc. Design Autom. Conf., pp. 439-445, June 1993.

[15] M. H. Schulz, K. Fuchs, and F. Fink, "Advanced Automatic Test Pattern Techniques for Path Delay Faults", Proc. Fault Tolerant Comp. Symp., pp. 4451, 1989.

[16] G. L. Smith, "Model for Path Delay Faults Based on Paths", Proc. Int'l Test Conf, pp. 342-349, 1985. 